\title{
Las expresiones de los estudiantes de instituciones de nivel superior en torno a las personas con discapacidad
}

\author{
Higher Education Students' Expressions with Respect to Disabled \\ People
}

\author{
Jesús García Reyes (*) jgarcia8207@gmail.com \\ (*) Universidad Nacional Autónoma de México \\ (Recibido: 27 de abril de 2017; Aceptado para su publicación: 30 de junio de 2017)
}

Cómo citar: García, J. (2019). Las expresiones de los estudiantes de instituciones de nivel superior en torno a las personas con discapacidad. Revista Electrónica de Investigación Educativa, 21, e10, 1-12. doi:10.24320/redie.2019.21.e10.1949

\section{Resumen}

La investigación es una aproximación al problema de la exclusión hacia las personas con discapacidad por parte de estudiantes de Instituciones de Educación Superior del país. Se elaboró un instrumento basado en la asociación de palabras para luego utilizar la técnica de redes semánticas naturales a una muestra de 519 estudiantes de nueve Instituciones de Educación Superior en México para conocer las expresiones sobre el tema de la discapacidad empleando una palabra generadora de uso cotidiano. Aunque los hallazgos evidencian aceptación aún prevalecen los estigmas y la desinformación que pueden llevar a la discriminación.

Palabras clave: Estudiantes, persona con discapacidad, educación, inclusión, exclusión.

\section{Abstract}

This study is an approach to the problem of exclusion of disabled people by students from the country's higher education institutions. An instrument was developed based on word association, in order to use the natural semantic network technique with a sample comprising 519 students from nine higher education institutions in Mexico, with the aim of gaining insight into their expressions with respect to disability by using an everyday trigger word. Although the findings reveal acceptance, stigma and misinformation still prevail, which may lead to discrimination.

Keyword: Students, person with disabilities, education, inclusion, exclusion. 


\section{Introducción}

Hablar de la Persona con Discapacidad (PCD) es referirse a un grupo en situación de vulnerabilidad. No se le puede considerar como una minoría porque es un grupo en crecimiento por diversas circunstancias, como son: accidentes, enfermedades, anomalías congenitas y lesiones temporales. Datos de la Organización Mundial de la Salud (OMS) señalan que el 15\% de la población mundial vive con algún tipo de discapacidad (OMS, 2011), por lo que el tema ha adquirido importancia en las agendas políticas nacionales e internacionales.

El Instituto Nacional de Estadística y Geografía $($ INEGI, 2016) reportó que 6\% de la población mexicana vive con alguna discapacidad: 6.2\% son mujeres y 5.7\% son hombres, en su mayoría jóvenes de entre 15 y 19 años. México sigue siendo un país en el que la inclusión de las personas con discapacidad es una realidad lejana porque las barreras culturales persisten y provocan rechazo al mantener distancia física y arquitectónica para una real convivencia. Consecuencia de esta situación es su baja incorporación en el sector laboral (donde casi el $85 \%$ realiza actividades en condiciones de informalidad) y su carente acceso a la educación: los mayores de 12 años que tienen alguna discapacidad carecen de escolaridad (26.4\%) y sólo un $17.9 \%$ concluye la primaria (Fuentes, 2015).

Dada esta situación, se han realizado estudios que analizan las percepciones y actitudes acerca de la integración de la PCD, como lo son la Encuesta Nacional de la Juventud 2010 y la Encuesta del Consejo Nacional para la Prevención de la Discriminación 2010, siendo estudios preámbulo de la investigación acerca de los jóvenes y la discriminación hacia los sectores vulnerables, que plantea la mirada que se tiene a su entorno y los desafíos para su reconocimiento.

En la encuesta del Consejo Nacional para Prevenir la Discriminación (Conapred, 2014), dos preguntas son relevantes y sugerentes para esta investigación porque refieren a la convivencia con distintos grupos, la primera tiene que ver con su relación próxima al preguntar: "¿Estarías dispuesto o no estarías dispuesto a permitir que en tu casa viviera una persona con discapacidad?". La respuesta mostraba una tendencia hacia la inclusión de la PCD por parte de los encuestados, pues 6 de cada 10 personas entablarían una relación dentro de su casa. La siguiente pregunta se relacionaba con el nivel de escolaridad y su convivencia con la PCD, al aumentar el nivel de conocimiento incrementa el nivel de inclusión.

\subsection{Investigaciones sobre la persona con discapacidad}

Respecto a los modelos y conceptos sobre la discapacidad, Padilla-Muñoz (2010) hace alusión a los mismos al mencionar: "Los grados son diferentes dependiendo si existe o no rehabilitación; así mismo, estarán influidos en gran parte si la persona vive en área rural o urbana, en un país desarrollado o en vías de desarrollo" (Padilla-Muñoz, 2010, p. 385). La autora menciona que en América Latina existen alrededor de 85 millones de personas que presentan algún tipo de discapacidad (10\% de la población mundial), el $80 \%$ vive en países pobres; asimismo, el número de PCD va en aumento por diversos, motivos: conflictos bélicos, minas antipersonas, desnutrición, VIH, enfermedades crónico-degenerativas, desastres naturales y accidentes, entre otros; tendencia que ha creado demandas en los servicios de salud (Padilla-Muñoz, 2010).

Por su parte, Castellanos y López (2013) trabajaron el concepto de discapacidad a partir de la experiencia social, donde se hace énfasis culturalmente a los rasgos negativos de la persona. La percepción cultural que se tiene del cuerpo y su relación con el pensamiento, comportamiento y modo de actuar se encuentra centrado principalmente en la imposición del prototipo de belleza actual, de modo que suele ser difícil la aceptación de los menos favorecidos.

En otro estudio, desde el enfoque de las Representaciones Sociales, Vargas (2014) muestra un panorama de la visión de los consejos locales de discapacidad sobre la PCD, la información, actitud y campo de representación del grupo se nutrió a partir de la experiencia vivida, la transmisión oral, las relaciones sociales y una idea cultural-religiosa, dando como resultado una variedad de respuestas tanto positivas, como neutras y negativas acerca de la PCD (Vargas, 2014). 
Las formas de pensar hacia la discapacidad han dependido históricamente de los estereotipos y prejuicios sociales de las imágenes predominantemente negativas sobre la idea de caridad y protección presentes en diversas culturas (Barnes, 1998). Sin embargo, las PCD han pasado de ser sujetos de asistencia a ser ciudadanos de participación dentro de su entorno, siendo la tendencia actual en países industrializados y en proceso paulatino en las demás sociedades.

En el caso de México prevalece una visión tradicional hacia la discapacidad. La sociedad desconoce su papel en las transformaciones necesarias para llevar a cabo la inclusión social de este grupo, perpetuando una orientación negativa y segregacionista (Bucio, 2013).

Los estudiantes dan cuenta de un significado compartido como grupo acerca de las PCD, expresandose su opinión acerca de cómo se presenta en la vida cotidiana. Las interacciones y expresiones suscitadas son reflejo de la experiencia vivida y su impacto ante un fenómeno determinado. La vida social se encuentra organizada por marcos de referencia aplicables mediante la interacción, presentación y actuación de cada uno de los miembros del grupo. De tal forma, que la PCD se presenta y representa su actividad ante otros en relaciones ordinarias, para ello, crea guías que controlan la impresión y las acciones ante los demás.

El trato con el otro no siempre es armonioso, en principio se encuentran las clasificaciones que pueden impedir una relación. El estigma es aquella marca que se asigna a las personas para desacreditarlas, separarlas de un grupo, ponerles apodos y hacer burlas soeces acerca de su condición (Goffman, 2008). Se presenta cotidianamente para evadir a los anormales, por su expresión diferente, por su nivel económico, por su visión del mundo, etc., y generalmente no se identifica con los rasgos comunes de los considerados normales -aquellos que no cuenten con dichas características son objeto de prejuicio y limitaciones para su plena aceptación social.

El uso cotidiano de la palabra discriminación se ha referido a cualquier acción que remita a apartar o excluir personas. Sin embargo, se debe agregar que es también la negación de uno o varios derechos, por parte de las instituciones o por las personas con algún poder (Rodríguez-Zepeda, 2006). En el caso de las $\mathrm{PCD}$, es un tema cotidiano, múltiples referencias y diferentes aristas simbólicas son parte de la apreciación de los llamados normales acerca de su persona. La visión errónea o los estigmas son prejuicios con respecto a una relación y en muchas ocasiones conllevan a la discriminación, cuando se le niegan derechos. La categorización estereotipada conlleva a una marca y exclusión para la convivencia.

\section{Método}

La muestra estuvo conformada por estudiantes de diferentes carreras y semestres de 9 Instituciones de Educación Superior (IES); se utilizaron dos instrumentos, un cuestionario con 60 preguntas y la asociación de palabras, que se aplicaron a 519 estudiantes, divididos en tres grupos:

1) 228 estudiantes de la Universidad Intercultural (UI) distribuidos de la siguiente manera: 72 de la Universidad Intercultural de Quintana Roo, 63 de la Universidad Intercultural del Estado de México y 93 de la Universidad Intercultural de Tabasco.

2) De las IES del centro del país se seleccionaron 169 estudiantes: 60 de la Escuela Benemérita Normal de Maestros, 55 de la Escuela Normal Superior de Maestros y 54 de la Escuela Superior de Educación Física.

3) De las IES del Bajío se contó con 122 estudiantes: 52 de la Universidad La Salle, 35 de la Universidad Tecnológica de León, y 35 de una Universidad privada de León.

Las entrevistas de la muestra fueron aleatoria, porque los entrevistados se buscaron al azar, de manera que se obtienen datos probabílistos. Se buscó la diversidad de estudiantes en diferentes espacios compartidos en la escuela. El instrumento que se utilizó para este texto es la asociación de palabra, que consistió en la recolección de las expresiones de los estudiantes en torno a las PCD. Se les presentó un término inductor, en este caso una palabra de uso cotidiano, como lo es "minusválido", un vocablo aceptado socialmente. Se mencionó este adjetivo por tener un mayor arraigo habitual. De este 
instrumento se construyeron categorías con base en las frecuencias que arrojaron el número de repeticiones de las palabras.

Para la construcción del siguiente instrumento de la red semántica natural se pidió al participante que jerarquizara sus respuestas de mayor a menor, siendo el número 1 el mayor y 3 el menor. Por último, se le pidió que justificara su respuesta. Para fundamentar la información y con el análisis de Reyes (1993), se elaboró una técnica de redes semánticas naturales, en donde el análisis de la asociación de palabras se trasladó a una base de datos y se sistematizó conforme a su jerarquización, para luego asignarle una ponderación, de manera que 1 equivale a 3, 2 a 2 y 3 a 1 . La suma de las palabras con el valor ponderado ofrece un peso semántico (PS) de éstas; por tanto, surgieron las más y las menos relevantes para los estudiantes.

Se realizó un acercamiento a la extensión de las expresiones de los estudiantes mediante un cuadro en el que se colocaron las palabras como fueron escritas previamente, para después depurar aquellas que contenían alguna falta de ortografía, las que aparecían en plural o términos semejantes, para obtener vocablos homogéneos lo más apegados al constructo de una red semántica (Reyes, 1993).

Posteriormente se organizaron las palabras de acuerdo a su jerarquía y se ordenaron tres tablas con su asignación de importancia, de mayor a menor. Por último, se les añadió el PS para seleccionar las 15 más significativas. Se examinó de forma detallada un acercamiento a la extensión de las expresiones que escribieron los estudiantes.

Se formó un conjunto de categorías para una interpretación más analítica de las respuestas obtenidas conforme a la teoría. Inició con dos categorías generales de inclusión y exclusión, como un primer acercamiento; sin embargo, durante el análisis se vio que existía un abanico más amplio de expresiones que no se podían aglutinar sólo en dos segmentos, por lo que construyeron otros conjuntos para una mayor comprensión e interpretación de las palabras.

Las redes semánticas naturales tienen como principio la búsqueda del significado que los individuos le otorgan desde su perspectiva común en el escenario donde se realiza la acción social, que está cargada de subjetividad y que es necesario comprender. En palabras de Goffman (2009), la interacción es simbólica porque se relacionan a partir de signos en común, lo que permite a los grupos forjar significados compartidos respecto a un objeto. Para fines de este artículo se presentan los resultados de las redes semánticas naturales y la construcción de categorías a partir de las respuestas.

En el proceso de análisis se construyeron dos categorías (Inclusión y Exclusión) y en cada una se agruparon subcategorías a partir de la connotación y PS de las palabras; de acuerdo con la relación semántica de la palabra se les asignó un nombre.

En la categoría de Inclusión se construyeron tres subcategorías analíticas:

1) Los Valores: identifican a las PCD a partir de sus cualidades y rasgos propios.

2) Los Atributos: son palabras positivas que denotan las características de las personas, sin cuestionar o enfatizar una situación positiva referente al grupo.

3) Los Derechos: se les sitúa en las expresiones con mayor aceptación porque consideran a las personas parte del grupo y reconocen su autonomía y bienestar mínimo.

Mientras en la categoría Exclusión se situaron dos subcategorías.

1) Las Particularidades: se colocaron los calificativos donde se señalan los rasgos singulares, distintivos y se clasificaron a partir de su condición (es la parte opuesta de los atributos porque hacen notar lo anormal o lo desviado y lo que está fuera de lo socialmente aceptado). 
2) El Estigma: las palabras que hacen hincapié en la marca de distinción y la barrera social que se le ha impuesto para la convivencia.

\section{Resultados}

El análisis de las redes semánticas consideró a las palabras con mayor PS como las expresiones más significativas en torno la persona con discapacidad, fue una primera exploración con los estudiantes de IES. Posteriormente, se buscó agrupar las respuestas en grupos a partir de términos semejantes a la inclusión y exclusión; por último, se crearon subcategorías para identificar de forma más precisa a la compresión y el reconocimiento de la persona, con respecto a aquellas palabras que mantiene un distanciamiento para la inclusión. De modo que los estudios de juventud y su relación con el entorno son de suma importancia para una sociedad moderna y diversa.

En la actualidad los jóvenes cuentan con diversos elementos que les permiten adquirir la información acerca de los temas actuales, los medios de comunicación, las redes sociales y los contenidos académicos son promotores de una cultura de la inclusión. Sin embargo, el efecto no es inmediato y existen particularidades que marcan al grupo, como es repetir patrones culturales negativos y una perpetración de la idea de normalidad, como parte de una cultura hegemónica.

La finalidad de este estudio es conocer las expresiones de los estudiantes entorno a la PCD. La muestra da evidencia de expresiones semejantes ante un fenómeno cotidiano y por tanto, su importancia para atenderlo. A continuación, se desarrollan los hallazgos obtenidos de la investigación.

\subsection{Categoría Inclusión}

En la vida cotidiana las personas establecen relaciones en espacios determinados, la fachada es la imagen o actividad que realiza las personas en un período continuo, ante un conjunto particular de observadores que poseen influencia sobre ellos. (Goffman, 2009). La palabra más relevante que asignaron los estudiantes fue discapacidad (134), comprendiendo la condición en la que vive la persona y su imagen. Véase Tabla I. 
Tabla I. Peso semántico de las palabras en la categoría Inclusión

\begin{tabular}{|c|c|}
\hline Palabra & $\begin{array}{c}\text { Peso } \\
\text { Semántico }\end{array}$ \\
\hline Discapacidad & 134 \\
\hline Persona & 85 \\
\hline Capacidad & 47 \\
\hline Igualdad & 38 \\
\hline Ayuda & 33 \\
\hline Respeto & 33 \\
\hline Aроуо & 32 \\
\hline Vida & 24 \\
\hline Fuerza & 23 \\
\hline Triste & 23 \\
\hline Especial & 22 \\
\hline Inteligencia & 21 \\
\hline Superación & 18 \\
\hline Trabajo & 17 \\
\hline Valentía & 17 \\
\hline Derechos & 15 \\
\hline Caminar & 14 \\
\hline Lucha & 14 \\
\hline Perseverancia & 14 \\
\hline Ser & 14 \\
\hline Autoestima & 13 \\
\hline Capaz & 13 \\
\hline Esfuerzo & 13 \\
\hline Oportunidad & 13 \\
\hline Capacidad & 12 \\
\hline Habilidad & 12 \\
\hline Atención & 11 \\
\hline Autosuficiente & 10 \\
\hline Competente & 10 \\
\hline Cuidado & 10 \\
\hline Emprendedor & 10 \\
\hline
\end{tabular}

\subsubsection{Subcategoría Valores}

Los valores se definen de manera general con la identificación de las personas; en este caso, el del grupo y cómo se presentan sus cualidades y rasgos propios del colectivo que los caracteriza frente a los demás (Goffman, 2009). Mostrar las cualidades de las personas es exponer la subjetividad de cada persona en su vida, y su propia identificación única.

Expresiones como: apoyo (32), ayuda (33), superación (18), lucha (14), perseverancia (14), oportunidad (13), esfuerzo (13), atención (11) y cuidado (10) demuestran la relación simbólica que se tiene sobre las personas que viven con alguna deficiencia; de forma positiva, las expresiones de los estudiantes relacionan una visión subjetiva hacia la condición individual a la que debe enfrentar día con día, denota información por parte de los jóvenes al conocer la terminología que se utiliza actualmente en relación con las PCD.

De manera que la expresión apoyo (32) demuestra que existe un conocimiento acerca del tema, muy probablemente la información fue adquirida a través de los medios de comunicación, carteles en las escuelas o cursos impartidos dentro de ellas, campañas gubernamentales y noticias al respecto son parte 
importante para la construcción del significado sobre la condición de la PCD (ver figura 1).

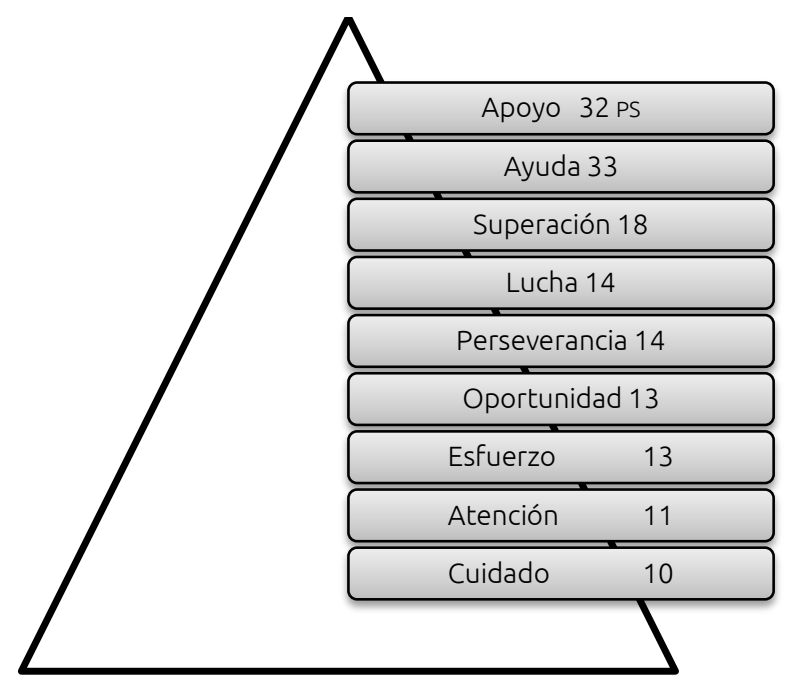

Figura 1. Palabras con su peso semántico en la subcategoría Valores

\subsubsection{Subcategoría Atributos}

Expresiones como: persona (88), vida (24), inteligencia (21), humano (17), ser (14), autoestima (13) capaz (13), habilidad (12), competente (10) y emprendedor (10) demuestran la unidad de una idea sobre las PCD, muestran también las cualidades de cada ser humano, lo que les permite ser incluidos en las actividades del colectivo, su desempeño no se cuestiona. Los atributos son la identidad personal y del grupo.

Las expresiones de los estudiantes señalan a los atributos como la imagen de las personas que viven una discapacidad y una oportunidad para incluirlas plenamente en una sociedad diversa. Las palabras en este segmento expresaron la condición más descriptiva de las personas, lo que implica una relación directa hacia el trato de con las PCD (ver figura 2).

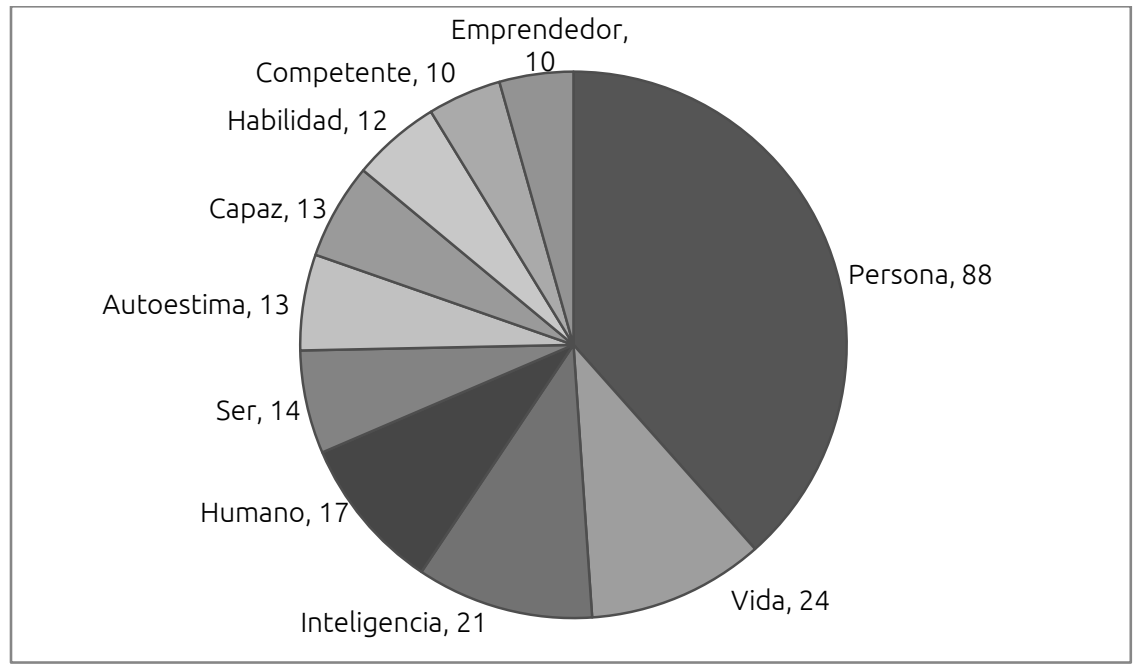

Figura 2. Palabras con su peso semántico en la subcategoría Atributos 


\subsubsection{Subcategoría Derechos}

Las palabras que se mencionaron son las que dan cuenta del compromiso social que se tiene con el grupo. Se distinguen expresiones de apertura social en comunidad y particularmente en los derechos que cada individuo cuenta y deben de ser respetados para convivir en armonía y de plena aceptación. Expresiones como: igualdad (38), respeto (33), educación (10) y derechos (10) señalan los elementos de importancia para una plena aceptación social de todos los miembros de la sociedad, de tal manera que las expresiones que se manifestaron demuestran conocimiento y concientización sobre lo que ocurre con los miembros de la comunidad (ver figura 3).

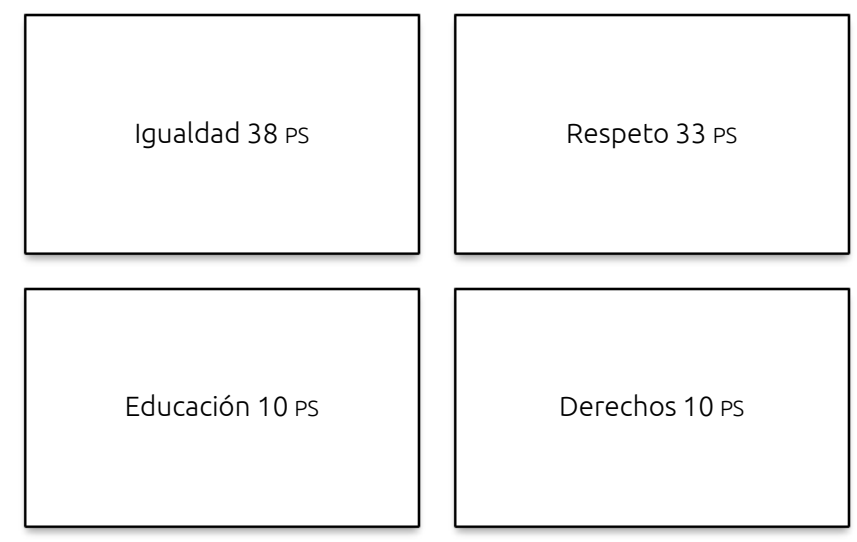

Figura 3. Palabras con su peso semántico en la subcategoría Derechos

\subsection{Categoría Exclusión}

El peso semántico de este grupo se encuentra alejado del núcleo de la red, lo que indica que es un grupo reducido que expresa la solidaridad y aceptación plena hacia las PCD. Aún hacen falta campañas, no sólo de información sino de sensibilización para que los estudiantes comprendan la situación en la que viven las PCD. Un elemento importante es su reconocimiento de la identidad personal al visibilizar la existencia de demás grupos sociales que conviven en una sociedad, al mencionar su rol en la sociedad. No obstante, es necesario ampliar el abanico de información y sensibilización, porque así se permitirá la aceptación plena de las PCD (ver tabla II). 
Tabla II. Palabras con su peso semántico en la categoría Exclusión

\begin{tabular}{l|c}
\hline \multicolumn{1}{c}{ Palabras } & $\begin{array}{c}\text { Peso } \\
\text { semántico }\end{array}$ \\
\hline Incapacidad & 56 \\
\hline Enfermo & 50 \\
\hline Diferente & 32 \\
\hline Silla de ruedas & 31 \\
\hline Inválido & 27 \\
\hline Deficiencia & 17 \\
\hline Inútil & 17 \\
\hline Dificultad & 15 \\
\hline Accidente & 14 \\
\hline Dependencia & 13 \\
\hline Necesidad & 12 \\
\hline Incompleto & 11 \\
\hline Lástima & 10 \\
\hline Rechazo & 10 \\
\hline Teletón & 10 \\
\hline Muletas & 9 \\
\hline Débil & 8 \\
\hline No camina & 8 \\
\hline Paralítico & 8 \\
\hline Estorbo & 7 \\
\hline Impotencia & 6 \\
\hline Limitación & 6 \\
\hline Lisiado & 6 \\
\hline
\end{tabular}

En este grupo se encuentran aquellas expresiones que describen los rasgos más visibles que caracterizan a las PCD. Mostrando la parte opuesta de los atributos y haciendo notar lo desviado, anormal y fuera de lo socialmente aceptado. La identidad deteriorada se encuentra en que las particularidades se aumentan y se segrega para mostrar los rasgos negativos de las personas (Goffman, 2008).

\subsubsection{Subcategoría Particularidades}

Expresiones como incapacidad (56), enfermo (50), silla de ruedas (31), diferente (32), necesidad (12), incompleto (11), paralítico (8), rechazo (10) y limitación (6) marcan una exclusión sistemática de las PCD, aun siendo una mención de las circunstancias en las que viven, la discriminación es una muestra del descarte de diversos grupos. De tal manera que expresiones como "enfermo, rechazo, limitación" son menciones de una situación de vida, pero en el trasfondo marcan una exclusión de baja intensidad, al ser descrito de forma segregada.

Expresiones simbólicas como "silla de ruedas" y "paralítico" muestran un rasgo descriptivo del defecto y su alejamiento con el llamado grupo de "normales". Mientras que vocablos como "necesidad" son atributos deseables para otorgar la caridad y comprensión ante la situación en la cual viven (ver tabla III). 
Tabla III. Palabras con su peso semántico en la subcategoría Particularidades

\begin{tabular}{l|c}
\hline Incapacidad & $\mathbf{5 6}$ \\
\hline Enfermo & 50 \\
Diferente & 32 \\
Silla de ruedas & 31 \\
Necesidad & 12 \\
Incompleto & 11 \\
Rechazo & 10 \\
Paralítico & 8 \\
Limitación & 6 \\
\hline
\end{tabular}

Nota: Elaboración propia.

\subsubsection{Subcategoría Estigma}

En la actualidad el término estigma ha cambiado y pasó de ser una mención o señalar a una persona que mostraba algún tipo de marca corporal -como solía usarse en la antigüedad-a una forma de distinción entre personas por medio del descrédito social. Ahora se puede decir que son motivo de estigma "las abominaciones del cuerpo, los defectos de carácter, los de carácter tribal, raza, [y] nación" (Goffman, 2008, p.14).

El uso actual es Persona con Discapacidad; por el contrario, llamar incompleto y relacionarlo con discapacitado es un estigma porque se refiere a que existen barreras para enfrentar los obstáculos de la vida diaria, como el de relacionarse e interactuar con los grupos de normales. Su PS es el mayor de la red (11), es decir, que los estudiantes lo relacionan con una limitación física, es un término de uso cotidiano aunque -como ya se señaló- se trata de un barrera social más que el impedimento de la persona para realizar determinadas actividades.

El fenómeno de la discriminación sigue latente en la sociedad actual, pero emerge una nueva mirada por parte de los estudiantes. Los vocablos con las que ellos asocian la palabra Inválido (27) son las palabras usadas indistintamente para describir a las PCD o la negación de un derecho. La discriminación es la negación u obstrucción de un derecho y comienza, en la mayoría de los casos, con el prejuicio o devaluación de las personas por ser consideradas inferiores.

El siguiente vocablo que aparece es inútil (17), continuando con los términos usados de forma habitual y que funcionan para identificar el estado de ánimo de la PCD, de nueva cuenta con una limitación. De igual forma, expresa la negación del acceso a la movilidad, indica rechazo hacia alguien que no cuenta con la capacidad de valerse por sí mismo. Prevalecen, en menor medida, el uso de términos que tratan de menospreciar, como lo demuestran el uso de deficiencia (17) y accidente (14) como representación de la información incipiente que brindan los medios de comunicación y los mensajes institucionales de anteriores gobiernos; se mantiene una imagen de separación al mostrar a personas incompletas o diferentes a la mayoría de la población. Sin embargo, aunque estos términos son comunes se trata de omitirlos en la nueva visión de la discapacidad que se fomenta desde las onG e instituciones públicas.

En ocasiones, los estereotipos o prejuicios funcionan para señalar a las personas, en el caso de palabras como dificultad (15), lisiado (6) y Teletón (10) es interesante observar que muchas de las palabras que los estudiantes emplean para estigmatizar son reflejo de la opinión de personas cercanas a ellos, como amigos, familiares o compañeros de escuela.

Las palabras que se relacionan con el estigma son las que de forma cotidiana se expresan acerca de las PCD, lo que indica la necesidad de buscar alternativas para sustituir dichas prácticas; mediante la información y sensibilización probablemente se erradiquen del vocabulario cotidiano (ver figura 4). 


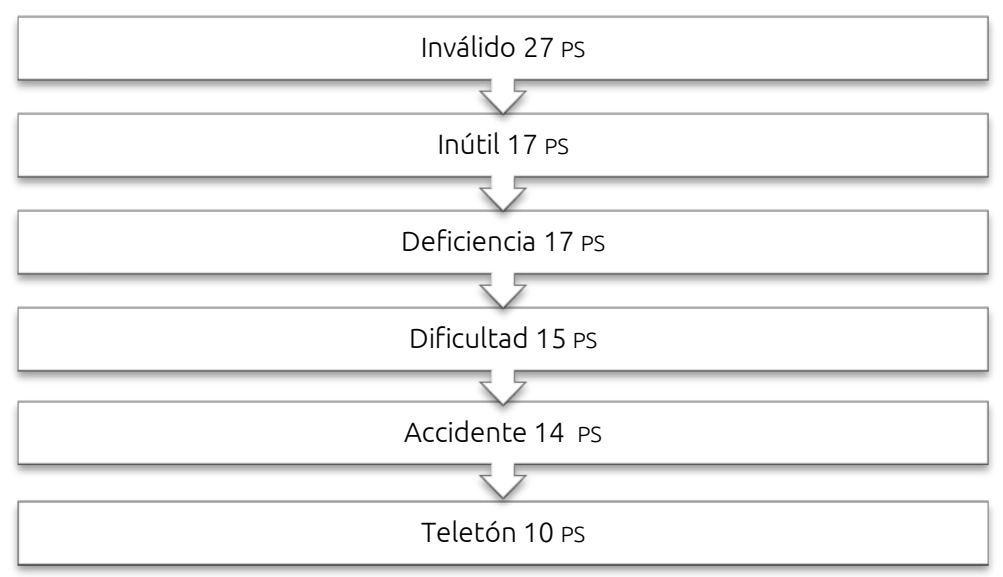

Figura 4. Palabras con su peso semántico de la subcategoría estigma

\section{Discusión y conclusiones}

Las respuestas de los estudiantes de nivel superior respecto a las PCD muestran su preocupación por mejorar las interacciones con ellos. Los jóvenes demostraron conocimiento de los temas actuales en cuanto a la aceptación de la diversidad; información y sensibilización van conformando el carácter dentro de las IES porque las relaciones con profesores y contemporáneos fortalecen los lazos aunado a la búsqueda de información y su preocupación por el entorno.

Las expresiones de los estudiantes respecto a las PCD muestran una preocupación sobre de lo que ocurre en su entorno, mostrando, en primer lugar, la situación en la que se encuentran las personas que viven con una deficiencia; en segundo término, su expresión hacia el respeto como persona dentro de una sociedad; y por último, muestran su preocupación hacia la condición de vulnerabilidad de la PCD al ratificar que viven una discriminación. El reto social en torno a la discapacidad se presenta en las expresiones de inclusión como un elemento para la construcción de una sociedad inclusiva, como principio básico de sentido y difusión de conocimiento para una sociedad heterogénea y que busca enriquecer la cultura ciudadana.

Sin embargo, la aceptación plena aún se encuentra en proceso de construcción porque hace falta mayor reconocimiento de los derechos fundamentales de las PCD, y dejar de ubicarlos como un grupo que necesita de la asistencia o de apoyo caritativo de las onG y de las personas que buscan dar protección al desamparado.

En el extremo de la red semántica se ubicaron las palabras que indicaron una exclusión hacia las PCD. Mantener una condición de vulnerabilidad es agravar la segregación que vive las PCD al excluirlas de manera generalizada de la vida social, económica y política, por la estigmatización directa o la falta de consideraciones hacia sus necesidades en el diseño de políticas, programas y servicios. Todavía hacen falta herramientas que permitan mayor comprensión y empatía para fomentar una cultura solidaria con las PCD, por ello es necesario buscar alternativas e involucrar a todos los actores sociales, para que permitan la construcción de una cultura ciudadana más plural y diversa en todos los espacios de la sociedad y que disminuya la desigualdad entre grupos. 


\section{Referencias}

Barnes, C. (1998). Las teorías de la discapacidad y los orígenes de la opresión de las personas discapacitadas en la sociedad occidental. En L. Barton (Comp.), Discapacidad y sociedad (pp. 59-77). Madrid: Morata.

Bucio, R. (diciembre, 2013). Construyendo un México incluyente. México Social, 41, 8-12. Recuperado de http://www.mexicosocial.org/index.php/2017-05-22-14-12-20/numeros-anteriores/item/426-numero-41diciembre

Castellano, F. y López, L. (2009). Discapacidad y cultura: desafío emergente en investigación. Avances en Enfermería, 1(27), 110-123. Recuperado de

https://revistas.unal.edu.co/index.php/avenferm/article/view/12960/13678

Consejo Nacional para prevenir la Discriminación. (2014). Estudio de parametría. México: Autor.

Instituto Nacional de Estadistica y Geografia. (2016). La Discapacidad en México, datos al 2014. Recuperado de http://internet.contenidos.inegi.org.mx/contenidos/productos/prod serv/contenidos/ espanol/bvinegi/productos/nueva estruc/702825090203.pdf

Fuentes, M.L. (marzo, 2015). Discriminación y pobreza. México Social, (56), 19-22. Recuperado de http://www.mexicosocial.org/index.php/2017-05-22-14-12-20/numeros-anteriores/item/738-numero-56marzo

Goffman, E. (2008). Estigma. La identidad deteriorada. Buenos Aires: Amorrortu.

Goffman, E. (2009). La presentación de la persona en la vida cotidiana. Buenos Aires: Amorrortu.

Organización Mundial de la Salud. (2011). Informe mundial sobre la discapacidad. Malta: Banco Mundial. Recuperado de http://www.who.int/disabilities/world report/2011/summary es.pdf?ua=1

Padilla-Muñoz, A. (2010). Discapacidad, contextos, conceptos modelos. International Law: Revista Colombiana de Derecho Internacional, 8(16), 381-414. Recuperado de

https://revistas.javeriana.edu.co/index.php/internationallaw/issue/view/937

Reyes, I. (1993). Las redes semánticas naturales, su conceptualización y su utilización en la construcción de instrumentos. Revista de Psicología Social y Personalidad, IX(1), 81-97.

Rodríguez-Zepeda, J. (2006). Un marco teórico para la discriminación. México: Conapred.

Vargas, R. (2014). Representaciones sociales de la discapacidad en los consejos locales de discapacidad de Bogotá: Revista Red Iberoamericana de Expertos en la Convención de los Derechos de las Personas con Discapacidad. Recuperado de http://www.repositoriocdpd.net:8080/handle/123456789/217 